\title{
An Obesity Dietary Quality Index Predicts Abdominal Obesity in Women: Potential Opportunity for New Prevention and Treatment Paradigms
}

\author{
Dolores M. Wolongevicz, ${ }^{1}$ Lei Zhu, ${ }^{2}$ Michael J. Pencina, ${ }^{2}$ Ruth W. Kimokoti, ${ }^{1}$ P. K. Newby, ${ }^{3}$ \\ Ralph B. D'Agostino, ${ }^{2}$ and Barbara E. Millen', 4 \\ ${ }^{1}$ Department of Family Medicine and the Graduate Medical Sciences Division, Boston University School of Medicine, \\ Boston, MA 02118, USA \\ ${ }^{2}$ Department of Mathematics, Boston University, Boston, MA 02215, USA \\ ${ }^{3}$ Departments of Pediatrics and Epidemiology, Boston University Schools of Medicine and Public Health, \\ Boston, MA 02118, USA \\ ${ }^{4}$ Boston Nutrition Foundation, Inc., Westwood, MA 02090, USA
}

Correspondence should be addressed to Barbara E. Millen, bmillen@bu.edu

Received 30 May 2009; Revised 15 October 2009; Accepted 25 November 2009

Academic Editor: Karen Charlton

Copyright (C) 2010 Dolores M. Wolongevicz et al. This is an open access article distributed under the Creative Commons Attribution License, which permits unrestricted use, distribution, and reproduction in any medium, provided the original work is properly cited.

\begin{abstract}
Background. Links between dietary quality and abdominal obesity are poorly understood. Objective. To examine the association between an obesity-specific dietary quality index and abdominal obesity risk in women. Methods. Over 12 years, we followed 288 Framingham Offspring/Spouse Study women, aged 30-69 years, without metabolic syndrome risk factors, cardiovascular disease, cancer, or diabetes at baseline. An 11-nutrient obesity-specific dietary quality index was derived using mean ranks of nutrient intakes from 3-day dietary records. Abdominal obesity (waist circumference $>88 \mathrm{~cm}$ ) was assessed during follow-up. Results. Using multiple logistic regression, women with poorer dietary quality were more likely to develop abdominal obesity compared to those with higher dietary quality (OR 1.87; 95\% CI, 1.01, 3.47; $P$ for trend $=.048$ ) independent of age, physical activity, smoking, and menopausal status. Conclusions. An obesity-specific dietary quality index predicted abdominal obesity in women, suggesting targets for dietary quality assessment, intervention, and treatment to address abdominal adiposity.
\end{abstract}

\section{Introduction}

More than $60 \%$ of adult females in the United States have abdominal obesity [1], a condition that independently predicts mortality [2], major morbidities [3], and metabolic risk factors [3] in women. Recent data suggest that waist circumference and the prevalence of abdominal obesity continue to increase [1]. Women may be at greater risk for abdominal obesity due to weight gain following pregnancy [4] and/or hormonal fluctuations at menopause, which shift body fat distribution from peripheral regions to the abdomen [5]. Current expert guidelines recommend initiating weight loss treatment in women whose waist circumference is $>88 \mathrm{~cm}$ (or BMI 25 to $29.9 \mathrm{~kg} / \mathrm{m}^{2}$ ) and who have two or more comorbidities, such as type 2 diabetes, cardiovascular disease (CVD), hypertension, or dyslipidemia [6]. Although abdominal fat decreases with weight loss, there is no consensus on an appropriate evidence-based preventive and treatment nutrition model to control abdominal obesity, and dietary interventions to sustain long-term weight loss ( $>1$ year) have not been identified. Many investigations of abdominal obesity measures and diet have focused on single-nutrients (e.g., alcohol, fat, protein) [7]; however, identification of the isolated nutritional effects is confounded in observational research by the coexistence of dietary factors in the foods we eat [8]. Examination of the total diet and nutrient intake patterns of individuals may provide better measures of diet exposure, helping identify individuals who 
may benefit from targeted nutritional risk interventions [9, 10]. Few studies have evaluated overall dietary quality using composite dietary indices/scores in relation to abdominal adiposity, [10-16] and the majority are cross-sectional investigations [11-15]. Further, none of the existing composite dietary quality indices/scores are based on a specific evidence basis for the most consistent dietary determinants of abdominal or peripheral obesity. The concept of a diseasespecific dietary quality index is unique and important since general indices without disease-specificity have not been predictive of all major chronic diseases in women [17, 18]. A dietary quality index/score that combines specific dietary determinants of obesity may be more helpful than existing approaches in identifying diet-obesity associations and may offer new insights into nutritional risk assessment, preventive intervention and treatment for abdominal obesity risk reduction. A nutrient-based approach, such as this, is appropriate because nutrient targeting influences patterns of food intake $[9,19,20]$, and the core of all US populationbased dietary guidelines and nutrition policy for maintaining health focus on achieving optimal nutrient intake with both nutrient and food-based messages.

This investigation prospectively examined the relationship between dietary quality and development of abdominal obesity in women, which has not been adequately addressed in current literature. We evaluated the ability of a novel obesity-specific nutritional risk score (ONRS) to predict development of abdominal obesity over 12 years in healthy women.

\section{Subjects and Methods}

2.1. Participants. The Framingham Study is a longitudinal population-based investigation that began in 1948 to study the progression of CVD. Detailed methods have been described elsewhere [21]. Briefly, cohort members were 5209 men and women, aged 28 to 62 years, from the town of Framingham, MA. In 1971, 5124 adult children and their spouses of the original cohort were invited to participate in the Framingham Offspring-Spouse Study (FOS). This second-generation cohort (2483 men and 2641 women) participated in clinical examinations every 4 years, on average. Each clinical visit, conducted according to a standardized protocol, consists of an updated, detailed medical history and a complete physical exam with laboratory and noninvasive diagnostic testing.

Of the 1956 women who attended the third examination (Exam 3, 1984-1988), only 1265 (65\%) had completed a 3 -day dietary record [9]. Of those 1265 women, 288 were not abdominally obese (waist circumference $\leq 88 \mathrm{~cm}$ ), aged 30 to 69 years, and presented without CVD (including coronary heart disease and stroke), cancer, diabetes or metabolic syndrome (MetS) risk factors at baseline (Exam 4, 1988-1992), and comprise the sample for these analyses. Waist circumference was first assessed at Exam 4; therefore, evaluation of baseline characteristics and covariates, except physical activity (Exam 2), also come from this exam. Diet exposure is Exam 3, not Exam 4, for these analyses because this is the collection time point for the 3-day dietary records; this approach is consistent with other published FOS research [10]. Follow-up was assessed through Exam 7 (1998-2001) for a total of 12 years.

All participants provided written informed consent. All protocols were approved by the Human Subjects Institutional Review Board of the Boston University Medical Campus and Boston Medical Center.

2.2. Diet Assessment and Nutritional Risk Score. Diet was assessed from 3-day dietary records completed at the Exam 3 clinic visit according to standardized research protocols $[22,23]$. Participants were instructed by a registered dietitian to record all foods consumed over 2 weekdays and 1 weekend day without deviation in their current eating behavior. To quantify portion sizes, participants were trained using a validated 2-dimensional pictorial food portion model [23]. Trained coders reviewed and coded the dietary records following formal protocols. Nutrient intake calculations were performed using the Minnesota Nutrition Data System software (version 2.6; Food Database 6A; Nutrient Database 23; Nutrition Coordinating Center, University of Minnesota, Minneapolis, MN). Three-day mean nutrient intake estimates were determined for each participant.

We previously validated our approach to composite nutrient risk score assessment in this cohort [9] and we used this approach to construct the obesity-specific nutritional risk score (ONRS). The new ONRS is based on dietary factors that have been shown to either promote or protectagainst general obesity [24-27]. Potential components of the nutritional risk score were identified by reviewing existing dietary composite indices $[9,28,29]$, as well as the extensive literature on diet and obesity in human studies. We selected eleven components to include in the ONRS: total energy $(\mathrm{kJ})$, energy density $(\mathrm{kJ} / \mathrm{g})$, carbohydrate (\% energy), protein (\% energy), total, monounsaturated, polyunsaturated, and saturated fats (\% energy), fiber $(\mathrm{g} / 4184 \mathrm{~kJ})$, calcium $(\mathrm{mg} / 4184 \mathrm{~kJ})$, and alcohol (\% energy).

The methodology used to calculate the ONRS is similar to that of our previously validated Framingham Nutritional Risk Score as described in the original validation of dietary patterns in the FOS cohort [9]. Ranking of individual nutrients in the score is based on the number of women in the sample where each nutrient is ranked from 1 (low risk) to 288 (high risk) for every woman with completed 3-day dietary records. Ranks are assigned so that a woman with a desirable nutrient intake level (protective nutrients) receives a lower rank while a woman with an undesirable nutrient intake level (risk-promoting nutrients) receives a higher rank. Energy, energy density, alcohol, and total, saturated, monounsaturated, and polyunsaturated fat intakes were ranked low to high, whereas protein, carbohydrate, fiber, and calcium intakes were ranked high to low. The mean ranks of each individual nutrient are used to calculate the overall nutritional risk score of each woman. These composite scores are then ranked and categorized into tertiles.

Monounsaturated and polyunsaturated fats were ranked as risk-promoting nutrients in contrast to some reports suggesting that they may be protective $[30,31]$. This was 
done because the majority of monounsaturated fat consumed by FOS women in this time-frame was derived from animal sources with higher saturated fat content rather than plant sources, and the polyunsaturated fat levels contained partially hydrogenated margarine rather than marine or plant oils.

2.3. Outcome Measure. The main outcome was development of abdominal obesity at any time point during follow-up. Abdominal obesity was defined according to the recommended waist circumference level for women $(>88 \mathrm{~cm})[6]$. Waist circumference was measured at clinic visits at the umbilicus with participants standing and the tape measure parallel to the floor, according to standard protocol.

2.4. Covariates. Metabolic and anthropometric measurements are routinely collected at clinic visits according to validated methods [32, 33]: systolic and diastolic blood pressure (duplicate measurements), fasting lipids [total cholesterol, high density lipoprotein (HDL), low density lipoprotein (LDL), and triglycerides], fasting glucose, and BMI (height and weight). The National Cholesterol Education Program's Adult Treatment Panel III cutoffs were used to evaluate each woman's MetS risk factors [34]. Women were considered to be free of MetS risk factors according to the following criteria: glucose $<110 \mathrm{mg} / \mathrm{dL}$, blood pressure $<130 /<85 \mathrm{mmHg}$, triglycerides $<150 \mathrm{mg} / \mathrm{dL}$, $\mathrm{HDL}>50 \mathrm{mg} / \mathrm{dL}$, and waist circumference $\leq 88 \mathrm{~cm}$.

Selected socio-demographic and behavioral characteristics are also assessed at clinic visits. Self-reported dietary behavior was evaluated using the Framingham Food Habit Questionnaire and included adherence to a modified diet (currently "on a diet") and usual weight pattern described as stable ( \pm 5 pounds) or fluctuating ( \pm 10 pounds) body weight. Other self-reported characteristics included age, smoking status, physical activity, parity, menopausal status, and use of hormone replacement therapy. Physical activity was assessed using a standardized questionnaire [35] at Exam 2 (1979-1983) and not at Exam 3; these values were used in the analyses consistent with published Framingham protocols [29].

2.5. Statistical Analysis. Age-adjusted mean levels of baseline characteristics and nutrient intakes were computed for each nutritional risk score tertile. The GLM procedure in SAS (analysis of covariance) was used to compute age-adjusted means for continuous variables. Logistic regression (SAS procedure LOGISTIC) was used to compute age-adjusted proportions for dichotomous variables. Bonferroni's correction was used to adjust for multiple comparisons in analyses of baseline characteristics and nutrient intakes. The primary research goal was to examine the association between dietary quality, assessed by the ONRS, and development of abdominal obesity. Stepwise multiple logistic regression was used to evaluate other variables that were related to abdominal obesity. After the stepwise regression, we constructed a final model to establish that the variables did not attenuate the ONRS-abdominal obesity relationship. The final, fixed model included the following variables: age (continuous), physical activity (continuous), menopausal status (yes/no) and smoking status (never, former, or current smokers). Metabolic, anthropometric, and demographic variables that did not differ at baseline according to nutritional risk score tertile were not identified as confounders and were thus not added to the model. Odds ratios (ORs) were calculated for each nutritional risk score tertile of the ONRS with the lowest tertile as the referent group. The $P$-value for trend was determined using the tertile groups of the ONRS in a continuous form. Alpha was set at 0.05 for main outcome statistical testing.

Secondary analyses adjusting for age, physical activity, menopausal status, and smoking status, were conducted to determine the relationship of individual nutrient risk score components to abdominal obesity. Intakes of each index component were ranked low to high and categorized into tertiles, and ORs were calculated for each intake tertile with the lowest tertile as the referent group. The $P$-value for trend was determined using the tertile groups of intake for each individual nutrient in a continuous form. Alpha was set at 0.05 for this set of secondary analyses.

All analyses were performed using SAS software (version 9.1; 2003, SAS Institute, Cary, NC).

\section{Results}

At baseline, women with higher dietary quality (i.e., lowest nutritional risk tertile) were significantly older and smoked less during their lifetimes (Table 1). These women did not differ across tertiles in other baseline characteristics. Their metabolic profiles reflect the health status of this diseasefree cohort at baseline; their blood pressure, total cholesterol, LDL, HDL, triglyceride, and blood glucose levels were within normal ranges.

Nutrient intakes differed according to dietary quality (Table 2). Relative to women with higher dietary quality (i.e., lowest nutritional risk tertile), women with the poorest dietary quality (i.e., highest nutritional risk tertile) had lower intakes of fiber, calcium, protein, and carbohydrate, higher energy-dense diets, and higher intakes of total energy, alcohol and total, saturated, polyunsaturated, and monounsaturated fats.

The overall incidence of abdominal obesity over 12 years was $\sim 52 \%(n=149$; Table 3$)$. The ONRS was directly related to abdominal obesity ( $P$ for trend $=.048$ ). In multiple logistic regression analyses adjusted for age, physical activity, menopause, and smoking status, women in the highest nutritional risk tertile were 1.87 (95\% CI, 1.01, 3.47) times more likely to become abdominally obese compared to those in the lowest tertile of nutritional risk.

In secondary analyses that explored all ONRS nutrients individually, carbohydrate intake was inversely associated, while monounsaturated fat intake was positively associated, with development of abdominal obesity ( $P$ for trend <.05). In multiple logistic regression analyses adjusted for age, physical activity, menopause, and smoking status, compared to the lowest tertile of intake, odds of becoming abdominally obese were lower in the highest tertile of carbohydrate intake 
TABLE 1: Baseline characteristics of 288 healthy women without abdominal obesity (waist circumference $\leq 88 \mathrm{~cm}$ ) in the Framingham Offspring-Spouse Study according to dietary quality ${ }^{1,2}$.

\begin{tabular}{|c|c|c|c|}
\hline & & cific Nutrition & \\
\hline & & & \\
\hline & Dietary Quality & & Dietary Quality \\
\hline & Tertile 1 & Tertile 2 & Tertile 3 \\
\hline Characteristic & $n=96$ & $n=96$ & $n=96$ \\
\hline Age (years) & $51.7(0.88)^{\mathrm{a}}$ & $48.0(0.88)^{\mathrm{b}}$ & $46.1(0.88)^{b}$ \\
\hline Weight $(\mathrm{kg})^{4}$ & $60.0(0.74)$ & $59.9(0.72)$ & $60.6(0.73)$ \\
\hline $\mathrm{BMI}\left(\mathrm{kg} / \mathrm{m}^{2}\right)$ & $22.9(0.23)$ & $22.6(0.23)$ & $23.0(0.23)$ \\
\hline $\begin{array}{l}\text { Waist Circumference } \\
(\mathrm{cm})\end{array}$ & $71.9(0.63)$ & $72.0(0.62)$ & $73.4(0.63)$ \\
\hline $\begin{array}{l}\text { Physical Activity } \\
\text { Index }{ }^{5}\end{array}$ & $37.5(0.65)$ & $37.4(0.64)$ & $36.2(0.65)$ \\
\hline Current Smoker (\%) & 10.4 & 12.5 & 19.8 \\
\hline Smoking (pack years) & $6.0(1.46)^{\mathrm{a}}$ & $6.8(1.40)^{\mathrm{a}}$ & $11.8(1.44)^{\mathrm{b}}$ \\
\hline Current Dieter (\%) & 94.7 & 94.7 & 94.6 \\
\hline $\begin{array}{l}\text { Fluctuating Weight } \\
(\%)\end{array}$ & 10.1 & 9.8 & 11.4 \\
\hline Postmenopausal (\%) & 59.4 & 35.4 & 36.5 \\
\hline $\begin{array}{l}\text { On Hormone } \\
\text { Replacement Therapy } \\
(\%)\end{array}$ & 9.4 & 9.5 & 6.3 \\
\hline Parity (\# of births) & $2.1(0.15)$ & $2.6(0.14)$ & $2.4(0.14)$ \\
\hline $\begin{array}{l}\text { Systolic Blood } \\
\text { Pressure }(\mathrm{mmHg})\end{array}$ & $111.6(1.03)$ & $110.3(1.00)$ & $110.9(1.01)$ \\
\hline $\begin{array}{l}\text { Diastolic Blood } \\
\text { Pressure (mmHg) }\end{array}$ & $71.8(0.72)$ & $70.3(0.70)$ & $70.9(0.71)$ \\
\hline $\begin{array}{l}\text { Total Cholesterol } \\
(\mathrm{mmol} / \mathrm{L})^{6}\end{array}$ & $5.11(0.09)$ & $4.98(0.08)$ & $5.05(0.09)$ \\
\hline $\begin{array}{l}\text { High Density } \\
\text { Lipoprotein } \\
\text { Cholesterol }(\mathrm{mmol} / \mathrm{L})\end{array}$ & $1.64(0.03)$ & $1.72(0.03)$ & $1.72(0.03)$ \\
\hline $\begin{array}{l}\text { Low Density } \\
\text { Lipoprotein } \\
\text { Cholesterol }(\mathrm{mmol} / \mathrm{L})\end{array}$ & $3.08(0.08)$ & $2.9(0.08)$ & $2.98(0.08)$ \\
\hline $\begin{array}{l}\text { Triglycerides } \\
(\mathrm{mmol} / \mathrm{L})^{7}\end{array}$ & $0.84(0.03)$ & $0.77(0.03)$ & $0.75(0.03)$ \\
\hline Glucose $(\mathrm{mmol} / \mathrm{L})^{8}$ & $4.75(0.04)$ & $4.77(0.04)$ & $4.78(0.04)$ \\
\hline
\end{tabular}

${ }^{1}$ Values are least squares means (SE) or percent. The GLM procedure in SAS (analysis of covariance) was used to obtain age-adjusted means for continuous variables and to identify subgroups that differed significantly. Logistic regression (SAS procedure LOGISTIC) was used to obtain age-adjusted proportions for dichotomous variables and to identify subgroups that differed significantly. Both sets of analyses used Bonferroni's correction for each variable.

${ }^{2}$ Values in a row with different superscript letters are significantly different from each other $(P<.05)$. Rows with no superscript letters indicate NS differences.

${ }^{3}$ The risk score was calculated from the consumption of 11 nutrients (protein, carbohydrate, fiber, calcium, alcohol, total fat, polyunsaturated fat, monounsaturated fat, saturated fat, energy density, and total energy), which were ranked for each woman in the sample.

${ }^{4}$ To convert $\mathrm{kg}$ to pounds divide by 0.454 .

${ }^{5}$ Physical Activity Index scores range from 24 (total bed rest) up to 120.

${ }^{6} \mathrm{To}$ convert $\mathrm{mmol} / \mathrm{L}$ cholesterol to $\mathrm{mg} / \mathrm{dL}$ divide by 0.0259 .

${ }^{7}$ To convert $\mathrm{mmol} / \mathrm{L}$ triglyceride to $\mathrm{mg} / \mathrm{dL}$ divide by 0.0113 .

${ }^{8}$ To convert $\mathrm{mmol} / \mathrm{L}$ glucose to $\mathrm{mg} / \mathrm{dL}$ divide by 0.0555 . 
TABLE 2: Baseline daily nutrient intake profiles of 288 healthy women without abdominal obesity (waist circumference $\leq 88 \mathrm{~cm}$ ) in the Framingham Offspring-Spouse Study according to dietary quality ${ }^{1,2}$.

\begin{tabular}{|c|c|c|c|}
\hline & & ecific Nutritional & \\
\hline & Higher & & Lower \\
\hline & Dietary Quality & & Dietary Quality \\
\hline & Tertile 1 & Tertile 2 & Tertile 3 \\
\hline Nutrient & $n=96$ & $n=96$ & $n=96$ \\
\hline Total Energy $(\mathrm{kJ})^{4}$ & $6258.8(205.9)^{\mathrm{a}}$ & $7124.5(201.7)^{\mathrm{b}}$ & $7247.9(204.2)^{b}$ \\
\hline $\begin{array}{l}\text { Energy Density } \\
(\mathrm{kJ} / \mathrm{g})^{5}\end{array}$ & $3.05(0.08)^{\mathrm{a}}$ & $3.77(0.08)^{\mathrm{b}}$ & $4.18(0.08)^{\mathrm{c}}$ \\
\hline Total Fat (\% energy) & $31.6(0.43)^{\mathrm{a}}$ & $37.7(0.42)^{b}$ & $44.2(0.43)^{\mathrm{c}}$ \\
\hline $\begin{array}{l}\text { Polyunsaturated Fat } \\
\text { (\% energy) }\end{array}$ & $6.4(0.29)^{\mathrm{a}}$ & $8.0(0.28)^{\mathrm{b}}$ & $9.4(0.29)^{\mathrm{c}}$ \\
\hline $\begin{array}{l}\text { Monounsaturated Fat } \\
(\% \text { energy })\end{array}$ & $11.1(0.18)^{\mathrm{a}}$ & $13.7(0.18)^{b}$ & $16.2(0.18)^{\mathrm{c}}$ \\
\hline $\begin{array}{l}\text { Saturated Fat (\% } \\
\text { energy) }\end{array}$ & $11.4(0.26)^{\mathrm{a}}$ & $13.2(0.26)^{b}$ & $15.4(0.26)^{\mathrm{C}}$ \\
\hline Alcohol (\% energy) & $2.9(0.57)$ & $3.5(0.55)$ & $4.0(0.56)$ \\
\hline Protein (\% energy) & $18.1(0.39)^{\mathrm{a}}$ & $16.1(0.36)^{b}$ & $15.9(0.37)^{\mathrm{b}}$ \\
\hline $\begin{array}{l}\text { Carbohydrate (\% } \\
\text { energy) }\end{array}$ & $49.4(0.65)^{\mathrm{a}}$ & $44.4(0.63)^{\mathrm{b}}$ & $37.1(0.64)^{c}$ \\
\hline Fiber $(\mathrm{g} / 4184 \mathrm{~kJ})$ & $14.9(0.57)^{\mathrm{a}}$ & $13.6(0.56)^{\mathrm{a}}$ & $11.1(0.56)^{b}$ \\
\hline Calcium (mg/4184 kJ) & $678.3(28.3)^{\mathrm{a}}$ & $685.8(27.7)^{\mathrm{a}}$ & $571.1(28.1)^{\mathrm{b}}$ \\
\hline $\begin{array}{l}1 \text { Values are least squares } \\
\text { and to identify subgroups } \\
{ }^{2} \text { Values in a row with diffe } \\
{ }^{3} \text { The risk score was calc } \\
\text { monounsaturated fat, satu } \\
{ }^{4} \text { To convert kJ to kcal divic }\end{array}$ & $\begin{array}{l}\text { e GLM procedure } \\
\text { gnificantly. This set } \\
\text { t letters are significe } \\
\text { he consumption o } \\
\text { gy density, and total }\end{array}$ & $\begin{array}{l}\text { e) was used to obta } \\
\text { correction for each } \\
\text { r }(P<.05) \text {. Rows w } \\
\text { bohydrate, fiber, } \\
\text { for each woman in }\end{array}$ & $\begin{array}{l}\text { ontinuous variables } \\
\text { cate NS differences. } \\
\text { olyunsaturated fat, }\end{array}$ \\
\hline
\end{tabular}

TABle 3: Development of abdominal obesity over 12 years in 288 healthy women in the Framingham Offspring-Spouse Study according to dietary quality ${ }^{1}$.

\section{Obesity-Specific Nutritional Risk Score ${ }^{3}$}

Higher

Dietary Quality

Tertile 1

$n=96$
Tertile 2

$n=96$
Lower

Dietary Quality

Tertile 3

$n=96$

\begin{tabular}{|c|c|c|c|c|}
\hline \multicolumn{3}{|l|}{ Abdominally obese } & \multirow[b]{2}{*}{$55(57.3)$} & $\begin{array}{l}\text { Overall } \\
\text { Incidence }\end{array}$ \\
\hline$n(\%)$ & $47(49)$ & $47(49)$ & & $149(51.7)$ \\
\hline Odds ratio $(95 \% \mathrm{CI})$ & & & & $P$ for Trend ${ }^{3}$ \\
\hline Age-adjusted & 1 & $1.20(0.67,2.16)$ & $1.86(1.01,3.41)$ & 0.044 \\
\hline Multivariate-adjusted $^{4}$ & 1 & $1.23(0.68,2.24)$ & $1.87(1.01,3.47)$ & 0.048 \\
\hline
\end{tabular}

${ }^{1}$ Abdominal obesity defined as waist circumference $>88 \mathrm{~cm}$.

${ }^{2}$ The risk score was calculated from the consumption of 11 nutrients (protein, carbohydrate, fiber, calcium, alcohol, total fat, polyunsaturated fat, monounsaturated fat, saturated fat, energy density, and total energy), which were ranked for each woman in the sample.

${ }^{3}$ The $P$-value for trend was determined using the tertile groups of the Obesity-Specific Nutritional Risk Score in a continuous form. Significance testing $P<.05$.

${ }^{4}$ Multiple logistic regression model adjusted for age, smoking, physical activity, and menopause. 
(OR 0.50; 95\% CI, 0.27, 0.92) and higher in the highest tertile of monounsaturated fat intake (OR 1.91; 95\% CI, $1.04,3.52)$

\section{Discussion}

We evaluated dietary quality and its relationship to abdominal obesity using an 11-nutrient obesity-specific composite dietary risk index/score developed in the FOS. The ONRS predicted development of abdominal obesity over 12 years in women, aged 30 to 69 years, who were free of disease and MetS risk factors at baseline. Women with eating habits of poorest dietary quality (i.e., highest nutritional risk) consumed diets that were significantly lower in protein, carbohydrate, fiber, and calcium, and higher in total calories, energy-density, total fat, and alcohol.

Our longitudinal findings support those of crosssectional studies investigating the association of a priori dietary quality indices/scores and abdominal adiposity [1113, 15]. For example, Fogli-Cawley et al. [13] found that adults in the highest quartile of the 2005 Dietary Guidelines Adherence Index (DGAI) had the lowest waist circumference compared to those in the lowest quartile $(90 \mathrm{~cm}$ versus $96 \mathrm{~cm}$; $P$ for trend across 2005 DGAI quintiles <.001). The 2005DGAI uses 20 criteria, each with a maximum value of 1 , reflecting key dietary recommendations in the 2005 Dietary Guidelines for Americans [36].

These results are also consistent with earlier research using the Framingham Nutritional Risk Score (FNRS), a 19-nutrient CVD-oriented nutritional risk index, which also predicted abdominal obesity in FOS women [10]. Compared to women with higher dietary quality (i.e., lowest nutritional risk), those with poorer dietary quality (i.e., highest nutritional risk) had more than a twofold risk of developing abdominal obesity (OR 2.3; 95\% CI, $1.2,4.3)$. An advantage of the ONRS, however, is that it incorporates new foci of research, such as energy density and evidence-based predictors of obesity-related outcomes (e.g., carbohydrate, protein, fat, fiber, calcium, and total calories). Synergistic mechanisms among nutrients in the ONRS may be modulating metabolic pathways that may lead to excess abdominal fat. The lower fat and energy-density combined with higher fiber and protein intakes in women consuming a higher quality diet may reduce circulating levels of glucose and free-fatty acids, which may prevent insulin resistance and/or high insulin levels, leading to decreased visceral fat storage [37-39].

The role of diet in abdominal fat accumulation, however, is not clearly established. The findings of single-nutrient analyses by Halkjaer et al. [40] did not show total energy or macronutrient intake as a predictor of five-year changes in waist circumference in adults. To our knowledge, we are among the first to prospectively examine development of abdominal obesity and nutrient intake patterns using an obesity-specific dietary quality index/score with a specific energy density component and other evidence-based predictors of obesity. The results of these analyses suggest that overall dietary quality influences abdominal adiposity independent of age, physical activity, smoking, and menopausal status. Composite dietary quality appears more informative than the single nutrient approach in determining risk of abdominal obesity. While the ONRS cannot be directly applied "as is", dietary quality and intakes of the nutrients that comprise the score could become a new focus as these results are translated into public policy and assessment, prevention and treatment recommendations for abdominal obesity. Specifically, future translational research would assess nutrient-related risk using the ONRS combined with an evaluation of established dietary patterns [9] to identify food- and nutrient-based targets for preventive intervention to help control abdominal weight gain. As this type of translational research progresses, we could then encourage that nutrition interventions be guided by use of both a composite nutrient index and dietary pattern approach.

In a recent randomized controlled clinical trial [41], a calorie-reduced diet modeled on the Dietary Approaches to Stop Hypertension (DASH) protocol resulted in a significantly greater weight loss and reduction in waist circumferences in overweight and obese women after 6 months $(-14 \mathrm{~kg}, P=.03$ and $-5 \mathrm{~cm}, P=.04$, resp.) than the control group $(+1 \mathrm{~kg}$, NS and $-1 \mathrm{~cm}$, NS, resp.). The DASH diet plan emphasizes fruits, vegetables, low-fat dairy, whole grains, poultry, fish, and nuts and is higher in calcium and magnesium and lower in total and saturated fat. It is important to note that longer term efficacy of this dietary protocol was not determined and that this protocol may be difficult for some individuals to follow for extended periods, particularly if it requires major changes in habitual eating behavior and is not compatible with personal preferences and tastes. A dietary quality approach that targets obesity-specific nutrients, as demonstrated by the ONRS, combined with approaches that are informed by habitual eating patterns offers an alternative methodology. This current research suggests that opportunities exist for a new intervention paradigm that promotes improved nutrient quality while retaining the beneficial aspects of the individual's established eating pattern.

Although our results suggest that higher intakes of polyunsaturated and monounsaturated fats may increase a woman's risk of abdominal weight gain, it is important to emphasize that the food sources of monounsaturated and polyunsaturated fats consumed by FOS women in the mid-1980s baseline time-frame were not derived from protective plant sources, but rather animal sources high in saturated fat and partially hydrogenated margarines potentially high in trans fat, respectively. The increased risk of abdominal obesity associated with saturated and trans fat has been demonstrated in animal [42] and human [43, 44] investigations. Further, current clinical trial literature has demonstrated a protective effect of monounsaturated and polyunsaturated fats derived from plant oils on central fat distribution $[45,46]$. If the ONRS were to be applied to more recent dietary data, it would seem important to alter the scoring of these fat subtypes to reflect current diets.

In addition, given that the scoring system is based on ranking within a study population, direct application of the ONRS is not advised at this time. Future translational research needs to determine an optimal method of ranking 
individuals in other populations. Before we would advocate a direct translation of this index, we would wish to conduct a formal translational research study similar to one we conducted with FOS food-based dietary patterns developed from cluster analyses $[9,47]$. Translational research of this nature was beyond the scope of the present study but the importance of carrying out such analytical research seems supported by our present findings.

The major strength of this study is the longitudinal design with long duration of follow-up and direct measurement of the outcome variable (i.e., waist circumference), as opposed to self-report. Additionally, the ONRS is nutrientbased, which is consistent with the foundation of the Dietary Guidelines for Americans [36] for determining adequacy of intake and overall dietary quality. Other strengths include calculation of dietary quality using energy-adjusted index components and consideration of a wide range of potential confounders in stepwise analyses with the final fixed model adjusted for age, physical activity, smoking, and menopausal status. In addition, our methodology of including only components associated with obesity in the ONRS potentially increased the diagnostic accuracy of the index. This is a methodological strength in dietary quality index construction that has recently been noted by Kourlaba and Panagiotakos [48]. And although our scoring system approach, in which components of the ONRS are weighted equally, is consistent with other dietary quality indices $[28,29]$, ongoing work is required to refine the use and construction of such indices.

We acknowledge our relatively small sample size. Our outcome was development of abdominal obesity; therefore, it was critical to exclude women with abdominal obesity and those with related risk factors at baseline. The fact that fewer than $25 \%$ of women in the FOS cohort met the inclusion criteria underscores the high prevalence of abdominal obesity and related metabolic risk factors. Despite the smaller number of women included in this investigation, we were able to demonstrate the predictive validity between a lower quality diet and abdominal obesity.

Another limitation is the use of 3-day dietary records which may introduce error as they have been reported to underestimate intake [49] and are subject to reporting bias. We attempted to partially correct for energy misreporting in that all nutrients are energy-adjusted in the index using the nutrient-density method (i.e., \% kcal or unit of nutrient $/ 4184 \mathrm{~kJ}$ ) as discussed in a recent systematic review [50]. Nonetheless, it is our view that in this lean sample of women at baseline, energy mis-reporting (notably overreporting) would likely have attenuated the results and led to a failure to detect a relationship between dietary quality and abdominal obesity. While it is also true that we cannot assess nutritional risk over time, intakes in the FOS cohort have been shown to be stable [51].

We also note the potential for bias in our sample since of the 1956 women who attended Exam 3, only 1265 completed a 3-day dietary record. Previously, we have compared women who completed the 3-day dietary records to those who did not [9]. Women who completed the records were older $(\sim 49$ years versus $\sim 47$ years), had lower BMIs (25 versus 26 ), and were more likely to be nonsmokers. Further, these results may not be generalizable to women outside of the FOS as $98 \%$ of these women are white and eating patterns and nutrient intakes may differ by ethnicity [52]. We further recognize the importance of socio-economic status; however, information on this and other potential confounders was not available for these analyses.

In conclusion, we identified a relationship between dietary quality assessed using an obesity-specific dietary quality index/score and accumulation of central body fat. An energy-adjusted, nutrient-based risk score, like the ONRS, identifies important components of nutritional risk assessment for abdominal obesity prevention in women and suggests that new opportunities exist for preventive nutrition intervention paradigms that target specific improvements in dietary quality in the context of the individual's nutrient intake pattern. As future research moves to a translational model, the nutrient components of interest can be translated into targeted food sources, which can be used to guide improvements by establishing guidelines for the counseling process and optimal approaches to dietary interventions. Strategies of this nature are crucial in planning and implementing programs for abdominal and peripheral obesity risk reduction and individualized treatment recommendations.

\section{Acknowledgments}

This research was supported by Grants R01-HL-60700 and R01-HL-54776 from the National Heart, Lung, and Blood Institute (NHLBI) and funding from the Department of Family Medicine and the Division of Graduate Medical Sciences, Boston University School of Medicine. The Framingham Study is supported by NIH/NHLBI N01-HC-25195. DMW was responsible for the concept and design of the experiment and prepared the manuscript. BEM provided overall direction to this research, contributed to writing of the manuscript, and overall critical review of reported intellectual content. LZ carried out the statistical analyses and critically reviewed the statistical content reported. MJP and RBD oversaw the statistical analyses and contributed to the interpretation of the data and critical review of the statistical content reported. PKN contributed to the statistical design and writing of the manuscript. RWK contributed to interpretation of the data and writing of the manuscript. None of the authors had personal or financial conflicts of interest.

\section{References}

[1] C. Li, E. S. Ford, L. C. McGuire, and A. H. Mokdad, "Increasing trends in waist circumference and abdominal obesity among U.S. adults," Obesity, vol. 15, no. 1, pp. 216224, 2007.

[2] C. Zhang, K. M. Rexrode, R. M. van Dam, T. Y. Li, and F. B. $\mathrm{Hu}$, "Abdominal obesity and the risk of all-cause, cardiovascular, and cancer mortality: sixteen years of followup in US women," Circulation, vol. 117, no. 13, pp. 1658-1667, 2008. 
[3] I. Janssen, P. T. Katzmarzyk, and R. Ross, "Body mass index, waist circumference, and health risk: evidence in support of current national institutes of health guidelines," Archives of Internal Medicine, vol. 162, no. 18, pp. 2074-2079, 2002.

[4] H. S. Kahn, L. M. Tatham, and C. W. Heath Jr., "Contrasting factors associated with abdominal and peripheral weight gain among adult women," International Journal of Obesity, vol. 21, no. 10, pp. 903-911, 1997.

[5] J. C. Lovejoy, "The influence of sex hormones on obesity across the female life span," Journal of Women's Health, vol. 7, no. 10, pp. 1247-1256, 1998.

[6] National Institutes of Health, "Clinical guidelines on the identification, evaluation, and treatment of overweight and obesity in adults-the evidence report," report NIH Pub. No.98-4093, National Heart Lung and Blood Institute, Bethesda, Md, USA, 1998, http://www.nhlbi.nihgov/ guidelines/obesity/ob_gdlns.htm.

[7] J. Halkjær, T. I. A. Sørensen, A. Tjønneland, P. Togo, C. Holst, and B. L. Heitmann, "Food and drinking patterns as predictors of 6-year BMI-adjusted changes in waist circumference," British Journal of Nutrition, vol. 92, no. 4, pp. 735-748, 2004.

[8] A. K. Kant, "Indexes of overall diet quality: a review," Journal of the American Dietetic Association, vol. 96, no. 8, pp. 785-791, 1996.

[9] B. E. Millen, P. A. Quatromoni, D. L. Copenhafer, S. Demissie, C. E. O'Horo, and R. B. D’Agostino, "Validation of a dietary pattern approach for evaluating nutritional risk: the Framingham Nutrition Studies," Journal of the American Dietetic Association, vol. 101, no. 2, pp. 187-194, 2001.

[10] B. E. Millen, M. J. Pencina, R. W. Kimokoti, et al., "Nutritional risk and the metabolic syndrome in women: opportunities for preventive intervention from the Framingham Nutrition Study," American Journal of Clinical Nutrition, vol. 84, no. 2, pp. 434-441, 2006.

[11] A. Haveman-Nies, K. L. Tucker, L. C. P. G. M. de Groot, P. W. F. Wilson, and W. A. van Staveren, "Evaluation of dietary quality in relationship to nutritional and lifestyle factors in elderly people of the US Framingham Heart Study and the European SENECA study," European Journal of Clinical Nutrition, vol. 55 , no. 10 , pp. 870-880, 2001.

[12] J. H. Ledikwe, H. Smiciklas-Wright, D. C. Mitchell, G. L. Jensen, J. M. Friedmann, and C. D. Still, "Nutritional risk assessment and obesity in rural older adults: a sex difference," American Journal of Clinical Nutrition, vol. 77, no. 3, pp. 551$558,2003$.

[13] J. J. Fogli-Cawley, J. T. Dwyer, E. Saltzman, et al., “The 2005 dietary guidelines for Americans and risk of the metabolic syndrome," American Journal of Clinical Nutrition, vol. 86, no. 4, pp. 1193-1201, 2007.

[14] S. A. McNaughton, K. Ball, D. Crawford, and G. D. Mishra, "An index of diet and eating patterns is a valid measure of diet quality in an Australian population," Journal of Nutrition, vol. 138, no. 1, pp. 86-93, 2008.

[15] S. A. McNaughton, D. W. Dunstan, K. Ball, J. Shaw, and D. Crawford, "Dietary quality is associated with diabetes and cardio-metabolic risk factors," Journal of Nutrition, vol. 139, no. 4, pp. 734-742, 2009.

[16] S. K. Gao, S. A. A. Beresford, L. L. Frank, P. J. Schreiner, G. L. Burke, and A. L. Fitzpatrick, "Modifications to the healthy eating index and its ability to predict obesity: the multiethnic study of atherosclerosis," American Journal of Clinical Nutrition, vol. 88, no. 1, pp. 64-69, 2008.

[17] M. L. McCullough, D. Feskanich, M. J. Stampfer, et al., "Diet quality and major chronic disease risk in men and women: moving toward improved dietary guidance," American Journal of Clinical Nutrition, vol. 76, no. 6, pp. 1261-1271, 2002.

[18] M. L. McCullough, D. Feskanich, M. J. Stampfer, et al., "Adherence to the Dietary Guidelines for Americans and risk of major chronic disease in women," American Journal of Clinical Nutrition, vol. 72, no. 5, pp. 1214-1222, 2000.

[19] B. E. Millen, P. A. Quatromoni, M. Pencina, et al., "Unique dietary patterns and chronic disease risk profiles of adult men: the Framingham Nutrition Studies," Journal of the American Dietetic Association, vol. 105, no. 11, pp. 1723-1734, 2005.

[20] M. S. Westerterp-Plantenga, M. J. W. Ijedema, and N. E. G. Wijckmans-Duijsens, "The role of macronutrient selection in determining patterns of food intake in obese and non-obese women," European Journal of Clinical Nutrition, vol. 50, no. 9, pp. 580-591, 1996.

[21] M. Feinleib, W. B. Kannel, and R. J. Garrison, "The framingham offspring study. Design and preliminary data," Preventive Medicine, vol. 4, no. 4, pp. 518-525, 1975.

[22] B. M. Posner, S. S. Martin-Munley, C. Smigelski, et al., "Comparison of techniques for estimating nutrient intake: the Framingham Study," Epidemiology, vol. 3, no. 2, pp. 171-177, 1992.

[23] B. M. Posner, C. Smigelski, A. Duggal, J. L. Morgan, J. Cobb, and L. A. Cupples, "Validation of two-dimensional models for estimation of portion size in nutrition research," Journal of the American Dietetic Association, vol. 92, no. 6, pp. 738-741, 1992.

[24] US Department of Health and Human Services and the US Department of Agriculture and Dietary Guidelines Advisory Committee, "The report of the Dietary Guidelines Advisory Committee on Dietary Guidelines for Americans, 2005," Tech. Rep., US Government Printing Office, Washington, DC, USA, 2004, http://www.health.gov/ dietaryguidelines/dga2005/report/default.htm.

[25] World Health Organization, "Obesity: preventing and managing the global epidemic," WHO Technical Report Series 894, World Health Organization, Geneva, Switzerland, 2000.

[26] World Health Organization and Food and Agriculture Organization of the United Nations, "Diet, nutrition and the prevention of chronic diseases," WHO Technical Report Series 916, World Health Organization, Geneva, Switzerland, 2003.

[27] R. P. Heaney, K. M. Davies, and M. J. Barger-Lux, "Calcium and weight: clinical studies," Journal of the American College of Nutrition, vol. 21, supplement 2, pp. 152S-155S, 2002.

[28] E. T. Kennedy, J. Ohls, S. Carlson, and K. Fleming, "The healthy eating index: design and applications," Journal of the American Dietetic Association, vol. 95, no. 10, pp. 1103-1108, 1995.

[29] P. A. Quatromoni, M. Pencina, M. R. Cobain, P. F. Jacques, and R. B. D'Agostino, "Dietary quality predicts adult weight gain: findings from the Framingham Offspring Study," Obesity, vol. 14, no. 8, pp. 1383-1391, 2006.

[30] N. Moussavi, V. Gavino, and O. Receveur, "Could the quality of dietary fat, and not just its quantity, be related to risk of obesity," Obesity, vol. 16, no. 1, pp. 7-15, 2008.

[31] L. S. Piers, K. Z. Walker, R. M. Stoney, M. J. Soares, and K. O'Dea, “The influence of the type of dietary fat on postprandial fat oxidation rates: monounsaturated (olive oil) vs saturated fat (cream)," International Journal of Obesity, vol. 26, no. 6, pp. 814-821, 2002.

[32] L. A. Cupples and R. B. D’Agostino, “Some risk factors related to the annual incidence of cardiovascular disease and death by using pooled repeated biennial measurements: Framingham heart study, 30-year follow-up," in The Framingham Study: 
an Epidemiological Investigation of Cardiovascular Disease, W. B. Kannel, P. A. Wolf, and R. J. Garrison, Eds., Department of Health and Human Services, National Institutes of Health, Washington, DC, USA, 1987.

[33] J. B. Meigs, R. B. D’Agostino Sr., P. W. F. Wilson, L. A. Cupples, D. M. Nathan, and D. E. Singer, "Risk variable clustering in the insulin resistance syndrome: the Framingham Offspring Study," Diabetes, vol. 46, no. 10, pp. 1594-1600, 1997.

[34] National Institutes of Health, "Third report of the National Cholesterol Education Program (NCEP) on detection, evaluation and treatment of high blood cholesterol in adults (Adult Treatment Panel III)," Final Report 02-5215, National Heart, Lung and Blood Institute, Bethesda, Md, USA, 2002, http://www.nhlbi.nih.gov/guidelines/cholesterol/ atp3_rpt.htm.

[35] W. B. Kannel and P. Sorlie, "Some health benefits of physical activity. The Framingham Study," Archives of Internal Medicine, vol. 139, no. 8, pp. 857-861, 1979.

[36] US Department of Health and Human Services and US Department of Agriculture, "Dietary Guidelines for Americans, 2005," guidelines, US Government Printing Office, Washington, DC, USA, 2005, http://www.health.gov/ dietaryguidelines/dga2005/document/default.htm.

[37] M. Krebs and M. Roden, "Nutrient-induced insulin resistance in human skeletal muscle," Current Medicinal Chemistry, vol. 11, no. 7, pp. 901-908, 2004.

[38] J. P. Girod and D. J. Brotman, "The metabolic syndrome as a vicious cycle: does obesity beget obesity?" Medical Hypotheses, vol. 60 , no. 4 , pp. 584-589, 2003.

[39] H. Schröder, "Protective mechanisms of the Mediterranean diet in obesity and type 2 diabetes," The Journal of Nutritional Biochemistry, vol. 18, no. 3, pp. 149-160, 2007.

[40] J. Halkjær, A. Tjønneland, B. L. Thomsen, K. Overvad, and T. I. A. Sørensen, "Intake of macronutrients as predictors of 5-y changes in waist circumference," American Journal of Clinical Nutrition, vol. 84, no. 4, pp. 789-797, 2006.

[41] L. Azadbakht, P. Mirmiran, A. Esmaillzadeh, T. Azizi, and F. Azizi, "Beneficial effects of a dietary approaches to stop hypertension eating plan on features of the metabolic syndrome," Diabetes Care, vol. 28, no. 12, pp. 2823-2831, 2005.

[42] K. Kavanagh, K. L. Jones, J. Sawyer, et al., "Trans fat diet induces abdominal obesity and changes in insulin sensitivity in monkeys," Obesity, vol. 15, no. 7, pp. 1675-1684, 2007.

[43] M. Toeller, A. E. Buyken, G. Heitkamp, G. Cathelineau, and B. Ferriss, "Nutrient intakes as predictors of body weight in European people with type 1 diabetes," International Journal of Obesity, vol. 25, no. 12, pp. 1815-1822, 2001.

[44] P. Koh-Banerjee, N.-F. Chu, D. Spiegelman, et al., "Prospective study of the association of changes in dietary intake, physical activity, alcohol consumption, and smoking with 9-y gain in waist circumference among 16587 US men," American Journal of Clinical Nutrition, vol. 78, no. 4, pp. 719-727, 2003.

[45] L. K. M. Summers, B. A. Fielding, H. A. Bradshaw, et al., "Substituting dietary saturated fat with polyunsaturated fat changes abdominal fat distribution and improves insulin sensitivity," Diabetologia, vol. 45, no. 3, pp. 369-377, 2002.

[46] J. A. Paniagua, A. Gallego de la Sacristana, I. Romero, et al., "Monounsaturated fat-rich diet prevents central body fat distribution and decreases postprandial adiponectin expression induced by a carbohydrate-rich diet in insulin-resistant subjects," Diabetes Care, vol. 30, no. 7, pp. 1717-1723, 2007.

[47] M. J. Pencina, B. E. Millen, L. J. Hayes, and R. B. D’Agostino, "Performance of a method for identifying the unique dietary patterns of adult women and men: the Framingham Nutrition
Studies," Journal of the American Dietetic Association, vol. 108, no. 9, pp. 1453-1460, 2008.

[48] G. Kourlaba and D. Panagiotakos, "The number of index components affects the diagnostic accuracy of a diet quality index: the role of intracorrelation and intercorrelation structure of the components," Annals of Epidemiology, vol. 19, no. 10, pp. 692-700, 2009.

[49] A. F. Subar, V. Kipnis, R. P. Troiano, et al., "Using intake biomarkers to evaluate the extent of dietary misreporting in a large sample of adults: the OPEN study," American Journal of Epidemiology, vol. 158, no. 1, pp. 1-13, 2003.

[50] K. Poslusna, J. Ruprich, J. H. de Vries, M. Jakubikova, and P. van't Veer, "Misreporting of energy and micronutrient intake estimated by food records and 24 hour recalls, control and adjustment methods in practice," The British Journal of Nutrition, vol. 101, supplement 2, pp. S73-S85, 2009.

[51] B. E. Millen, M. Pencina, R. Kimokoti, and R. B. D’Agostino, "The Framingham Nutrition Studies: insights into weight history, dietary patterns, obesity prevention, and risk reduction," Tech. Rep., Department of Health and Human Services, National Institutes of Health, National Heart, Lung, and Blood Institute. Predictors of Obesity, Weight Gain, Diet, and Physical Activity Workshop, Bethedsa, Md, USA, August 2004, http://www.nhlbi.nih.gov/meetings/workshops/ predictors/abstracts/millen.htm.

[52] A. K. E. Wirfält and R. W. Jeffery, "Using cluster analysis to examine dietary patterns: nutrient intakes, gender, and weight status differ across food pattern clusters," Journal of the American Dietetic Association, vol. 97, no. 3, pp. 272-279, 1997. 


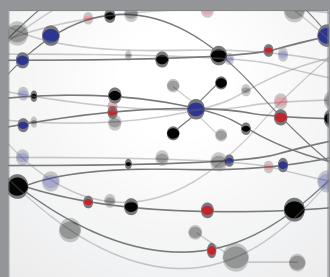

The Scientific World Journal
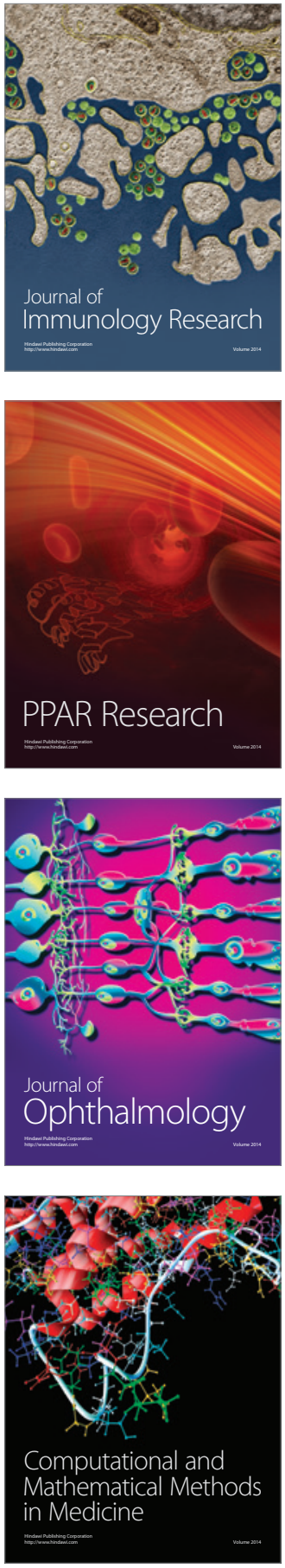

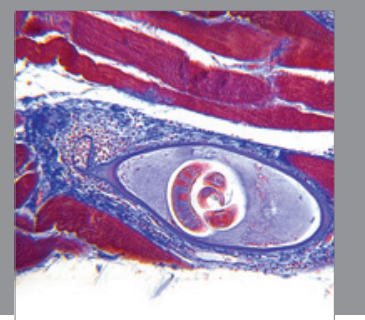

Gastroenterology

Research and Practice
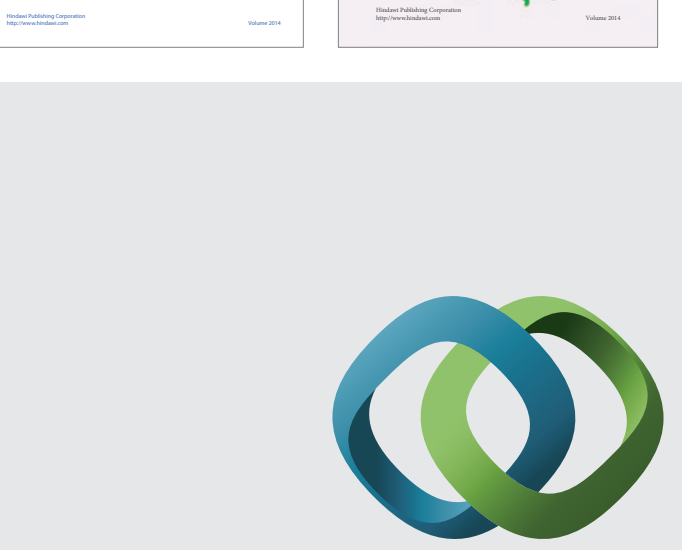

\section{Hindawi}

Submit your manuscripts at

http://www.hindawi.com
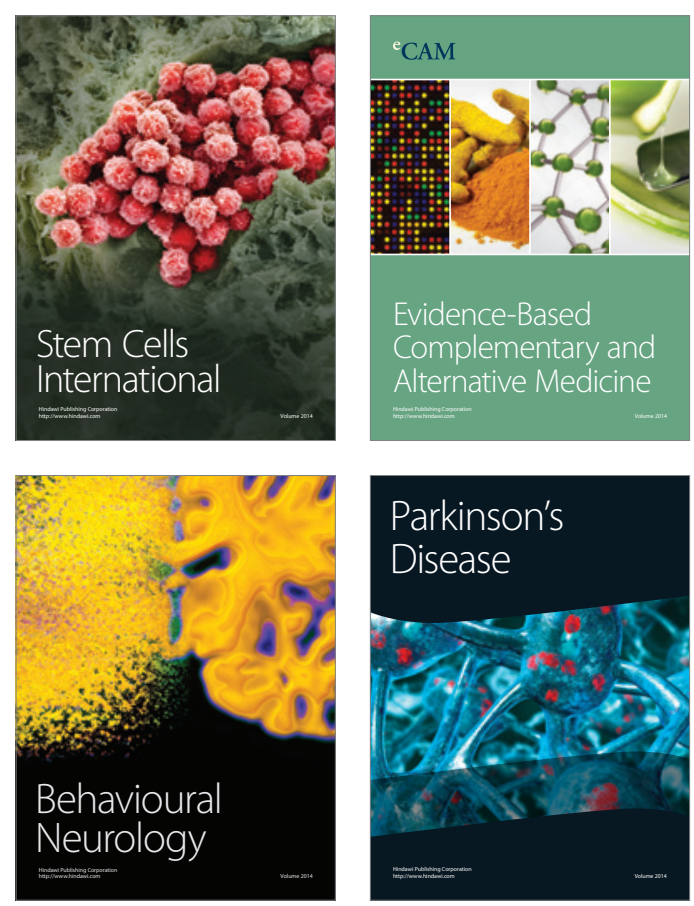

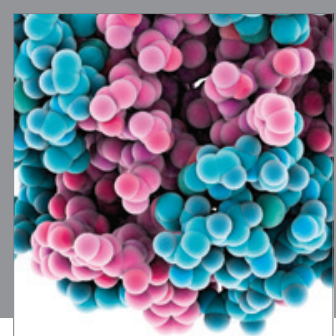

Journal of
Diabetes Research

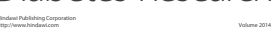

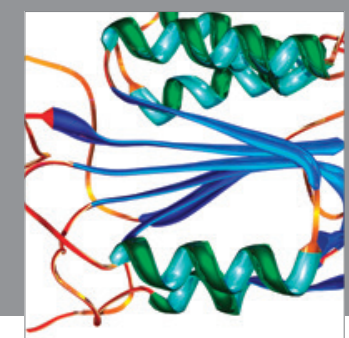

Disease Markers
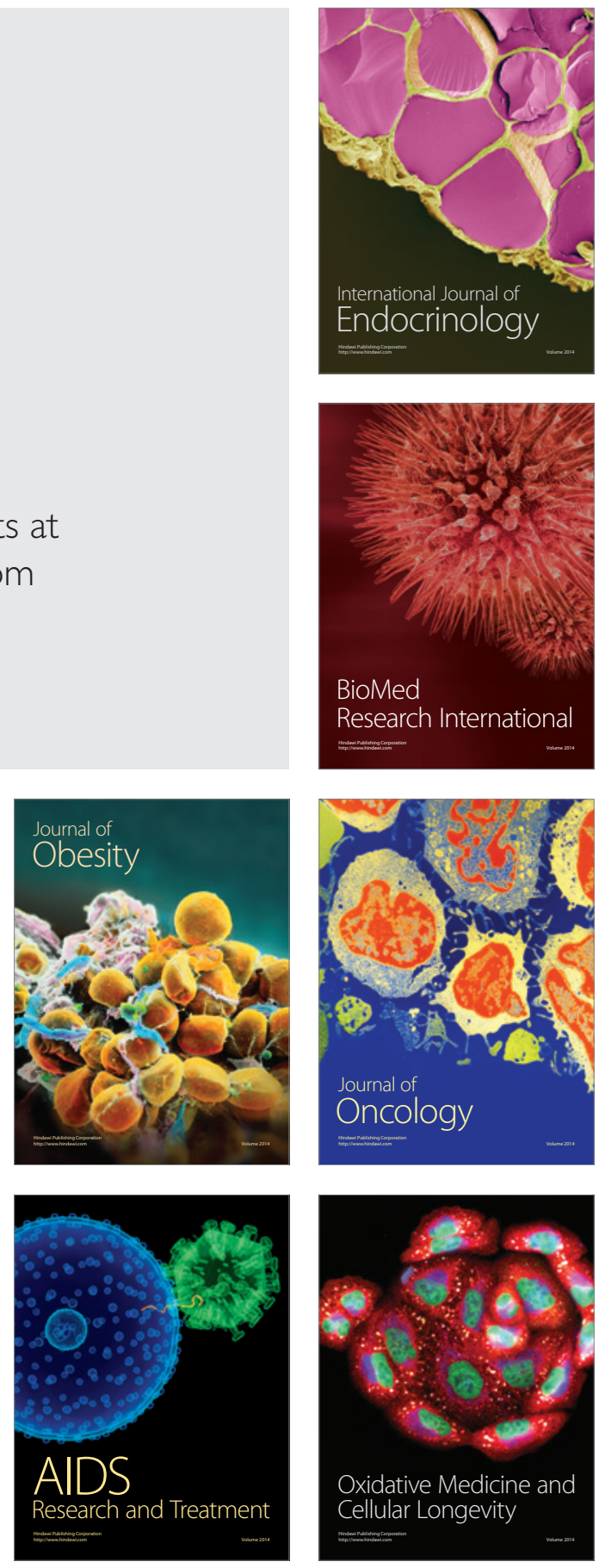